\title{
Diseño de equipos de contacto sólido-líquido a elevadas presiones en el procesamiento de la biomasa
}

\author{
Martín Enrique Durán García y y Ricardo Alejandro Ruiz Navas²
}

\section{Resumen}

La biomasa de tipo agrícola, en especial desechos de semillas y conchas de frutas, con fines utilitarios en la industria cosmética y de conversión de energía, representa un tema de interés que permite mitigar el pasivo ambiental generado por estos desechos y proporcionar una excelente materia prima para el procesamiento correspondiente en el parque industrial del área. Las tecnologías asociadas a los equipos de contacto sólido - líquido a elevadas presiones para el procesamiento de la biomasa comprenden el enfoque de ésta investigación, específicamente el estudio teórico de los principales aspectos que rigen el comportamiento hidráulico y las premisas de diseño existente en los equipos de contacto sólido-líquido destinados a la extracción de aceites esenciales de materias primas vegetales provenientes de la mandarina y durazno. En cuanto al modelo hidráulico empleado para modelar las baterías de difusión está representado por el criterio de estabilidad y la ley de Darcy, donde la caída de presión es lineal en función del caudal de solvente para una carga determinada. El tiempo de residencia está determinado por el tiempo de lavado y de drenado del solvente, y se encuentra entre 30 y 120 minutos de extracción. En el tamaño de la partícula, la reducción del mismo incide en un mejor desempeño del proceso, puede resultar costoso, lo que se requiere calcular el tamaño óptimo que ofrezca la máxima relación beneficio-costo. El estudio representa un aporte en la transformación de estos desechos a través de los equipos de contacto sólido - líquido a condiciones supercríticas.

Palabras clave: Biomasa Agrícola, Equipos de Contacto Sólido - Líquido, Extracción Supercrítica, Aceites Esenciales.

1 Magíster en Ingeniería de Sistemas, Ingeniero Químico. Profesor Agregado de la Universidad

Simón Bolívar e Investigador. Estudiante del Doctorado en Ingeniería Química. Universidad Simón Bolívar, Venezuela.

2 Ingenierio Químico. Estudiante de la Maestría de Ingeniería Química. Ayudante de Investigación. Universidad Simón Bolívar, Venezuela. 


\begin{abstract}
Type agricultural biomass, especially waste from seeds and shells of fruit, with utilitarian purposes in the cosmetics industry and energy conversion, is a topic of interest that can mitigate the environmental liabilities generated by these wastes and provide excellent raw material for the corresponding processing in the industrial park area. The technologies associated with solid contact equipment - a high pressure fluid processing biomass comprise the focus of this research, specifically the theoretical study of the main aspects that govern the hydraulic behavior and existing design premises equipment solid-liquid contact for the extraction of essential oils from plant materials tangerine and peach. As the hydraulic model used to model diffusion battery is represented by the stability criteria and Darcy's law, where the pressure drop is linear function of the flow of solvent for a given load. The residence time is determined by the washing time and the solvent drained, and is between 30 and 120 minutes of extraction. In the particle size, reducing it affects a better performance of the process can be costly, which is required to calculate the optimal size that provides the maximum benefit-cost ratio. The study represents a contribution to the transformation of these wastes through solid contact teams - liquid at supercritical conditions.
\end{abstract}

Key words: Biomass Agricultural Equipment Contact Solid - liquid, supercritical extraction, Essential Oils. 


\section{Introducción}

Un arreglo de compuertas lógicas programables (FPGA, Field Programmable Gate Array) es un dispositivo semiconductor que contiene bloques de lógica reprogramable dispuestos para la implementación de funcionalidades personalizadas en hardware, desarrolladas en tareas de cómputo digital en software y compiladas en un archivo de configuración (bitstream) que contiene información acerca de las conexiones que deben ser realizadas internamente. Asimismo, estos dispositivos son reconfigurables por lo que toman una nueva lógica cuando se compilan con una configuración diferente, también poseen un buen rendimiento que permite maximizar cada ciclo de reloj, además sobresalen sus precios factibles y su fiabilidad se presenta como ventaja fundamental al momento de poner en ejecución un proyecto, ya que mientras las herramientas software ofrecen un entorno de programación, los circuitos de un FPGA son una implementación segura de la puesta en marcha de un programa.

\section{Método}

El cumplimiento del objetivo de este trabajo requiere de un conjunto de actividades a realizarse que deben cumplir con las características generales de la investigación. El método utilizado es analítico según Bunge (1981) y Hurtado (2012), y donde se intenta comprender las situaciones en términos de las relaciones entre sus componentes, descubrir cada elemento e identificar las sinergias menos evidentes de los eventos analizados.

En la investigación analítica el resultado es la identificación de elementos no visibles o evidentes a los que no puede llegarse con una sencilla descripción (Hurtado, 2012). En esta investigación se llega al estadio de lograr objetivos complejos y se reinterpreta los eventos para llegar a conclusiones de diversa índole. A partir de los eventos a analizar se dispone de las fases del método analítico las cuales son cuatro, con el fin último de establecer criterios claros de análisis que permiten descubrir los elementos no visibles en el proceso investigativo planteado:

a.Definición de los eventos. Fase corresponde a la definición de los aspectos fundamentales y principios básicos que rigen el comportamiento hidráulico y las premisas de diseño existente en los equipos de contacto sólido-líquido destinados a la extracción de aceites esenciales de materias primas vegetales provenientes de la mandarina y durazno.

b.Construcción de la Matriz de Análisis. En esta fase se definen los principios hidráulicos, el tiempo de residencia del proceso y tamaño de partícula de la biomasa alimentada en el proceso de extracción sólido líquido a elevadas presiones. 
c. Aplicación de la Matriz de Análisis. En esta fase se adaptan y aplican los principios básicos del sistema sólido líquido al proceso industrial de procesamiento de la biomasa a presiones elevadas.

d.Análisis y conclusiones. Aquí se explican las implicaciones arrojadas por las principios y supuestos teóricos, de manera que se pueda comprender el diseño de estos equipos de contacto sólido líquido en el procesamiento de la biomasa.

Estas fases se desarrollan longitudinalmente durante toda el proceso de análisis, específicamente la fase construcción de eventos corresponde a los referentes teóricos y sus implicaciones presentadas previamente. En cuanto a la construcción y aplicación de la matriz de análisis constituye una herramienta fundamental que permite alcanzar los resultados que presentan las implicaciones encontradas al correlacionar el diseño de estos equipos con el procesamiento de este tipo de biomasa. La última fase está asociada al análisis de resultados que conducirán a las conclusiones.

\section{Análisis y Resultados}

\section{Heurísticas documentadas para diversos tipos de equipos de extracción}

En los equipos de extracción sólido-líquido principalmente se utilizan equipos de contacto continuo multietapas y en operación a contracorriente, según lo señala (Spaninks, 1979), (Walas, 1990), (Gamse, 2001), (Kemper, 2005), (Caldas, 2012), (González y Yánez, 2012), (Autor, 2014b), (Gorrití, 2015) y (Jaramillo, 2015). El aspecto operacional que más dificulta el diseño de éstos equipos radica en el manejo de los residuos y desechos sólidos o refinado remanente (Walas, 1990), (Caldas, 2012), (González y Yánez, 2012), (Autor, 2014b), (Gorrití, 2015), (Jaramillo, 2015). En la bibliografía prácticamente existen pocos indicios de datos o métodos heurísticos definidos para el diseño de los equipos de extracción sólido-líquido que operan de manera continua. Sin embargo, existe información de los parámetros de diseño más relevantes de la mayoría de los equipos diseñados y patentados a nivel industrial. Algunos de esos parámetros se presentan en la Tabla 1.

Tabla 1. Principales parámetros de diseño de los equipos comerciales de extracción sólidolíquido (Spaninks, 1979), (Walas, 1990), (Caldas, 2012) y (Gorrití, 2015).

\begin{tabular}{|c|c|c|c|}
\hline Extractor & Modelo & Volumen $(\mathrm{m} 3)$ & Capacidad $(\mathrm{m} 3 / \mathrm{h})$ \\
\hline Podbielniak & E48 & 0,925 & 113,5 \\
\hline Quadronic & Hiatchi 4848 & 0,9 & 72 \\
\hline$\alpha$-Laval & ABE 216 & 0,07 & 21 \\
\hline UPV & & & 6 \\
\hline
\end{tabular}




\begin{tabular}{|c|c|c|c|}
\hline Luwesta & EG 10006 & & 5 \\
\hline Robatel SGN & LX6 70NL & 0,072 & 3,5 \\
\hline Robatel BXP & BXP 800 & 0,220 & 50 \\
\hline Westfalia & TA 15007 & 0,028 & 30 \\
\hline SRL/ANL & & 0,003 & 0,05 \\
\hline MEAB & SMCS-10 & 0,00012 & 0,3 \\
\hline Extractor & Modelo & RPM & Ubicación del motor \\
\hline Podbielniak & E48 & 1600 & De lado \\
\hline Quadronic & Hiatchi 4848 & 1500 & De lado \\
\hline -Laval & ABE 216 & 6000 & Tope \\
\hline UPV & & 1400 & Fondo \\
\hline Luwesta & EG 10006 & 4500 & Fondo \\
\hline Robatel SGN & LX6 70NL & 1600 & Tope, de lado \\
\hline Robatel BXP & BXP 800 & 1000 & Tope \\
\hline Westfalia & TA 15007 & 3500 & Tope \\
\hline SRL/ANL & & 3500 & Tope \\
\hline MEAB & SMCS-10 & 2200 & Fondo \\
\hline Extractor & Modelo & Potencia del Motor (KW) & Diámetro (m) \\
\hline Podbielniak & E48 & 24 & 1,2 \\
\hline Quadronic & Hiatchi 4848 & 55 & 1,2 \\
\hline$\alpha$-Laval & ABE 216 & 30 & \\
\hline UPV & & 14 & \\
\hline Luwesta & EG 10006 & & \\
\hline Robatel SGN & LX6 70NL & & 1,3 \\
\hline Robatel BXP & BXP 800 & 15 & 0,8 \\
\hline Westfalia & TA 15007 & 63 & 0,7 \\
\hline SRL/ANL & & & 0,1 \\
\hline MEAB & SMCS-10 & & \\
\hline
\end{tabular}

Como puede observarse en la tabla 1 , se presentan 10 modelos de extractores sólido-líquidos que operan de manera continua a escala industrial. Básicamente su configuración está compuesta por un motor que hace girar el lecho de partículas distribuido en compartimientos y su potencia varía de acuerdo a la carga o capacidad. Se observa que la carga varía de 0,3 a $113,5 \mathrm{~m} 3 / \mathrm{h}$ de capacidad y la potencia varía de 14 a $63 \mathrm{KW}$. Es importante destacar que estas premisas de diseño (respecto a equipos existentes) puede fungir como punto de partida en la selección de bases y criterios de diseño para éste tipo de equipos de proceso. 


\section{- Parámetros de diseño típicos empleados en otros equipos de contacto continuo sólido-líquido}

El transporte continuo de sólidos en contracorriente a la entrada de solvente es empleado en muchas configuraciones de batería de equipos de extracción, incluyendo tornillos sinfín, cintas perforadas y elevador de cangilones. En los equipos de cintas perforadas, generalmente se emplean alturas de lecho de sólido de 3 a 4 pies, mientras el solvente fresco se aplica en relaciones que varían de 1/5 a $1 / 3$ de la distancia a la descarga y son transportadas por bombas que operan en contracorriente en todo el trayecto del material.

Equipos tales como el elevador de cangilones, posee alturas comprendidas entre los 40-60 pies y manejan en promedio una capacidad de $50 \mathrm{Ton} / \mathrm{h}$ consumiendo una potencia de 1-2 HP. Los cangilones son compartimientos cuyo fondo generalmente perforado, donde generalmente el extracto drena hacia el fondo del equipo.

Otra de las operaciones relevantes se lleva a cabo en los extractores tipo Bonotto. Dicho equipo opera a contracorriente en un número determinado de platos en un arreglo vertical, los cuales están provistos de unos dispositivos denominados "scrapers" que permiten transportar los sólidos mientras van drenando por los platos. El solvente es cargado en el tope de éste Contactor y sale junto a la corriente de solvente por el tope del mismo, y los sólidos remanentes de extraen mediante un tornillo sinfín.

Los parámetros y premisas de diseño ubicados en la bibliografía (Spaninks, 1979), (Walas, 1990), (Gamse, 2001), (Kemper, 2005), (Caldas, 2012) y (Jaramillo, 2015), corresponde a lo más cercano a las "Heurísticas" relacionadas directamente a éste tipo de equipos. Prácticamente no existe en la bibliografía una metodología de diseño explícita para éste tipo de equipos, tal cual existe por ejemplo para otros equipos de proceso tales como: torres de destilación-absorción, intercambiadores de calor, evaporadores, entre otros.

A continuación se muestran en las figuras 1,2, 3 y 4 los equipos mencionados en éste punto. 


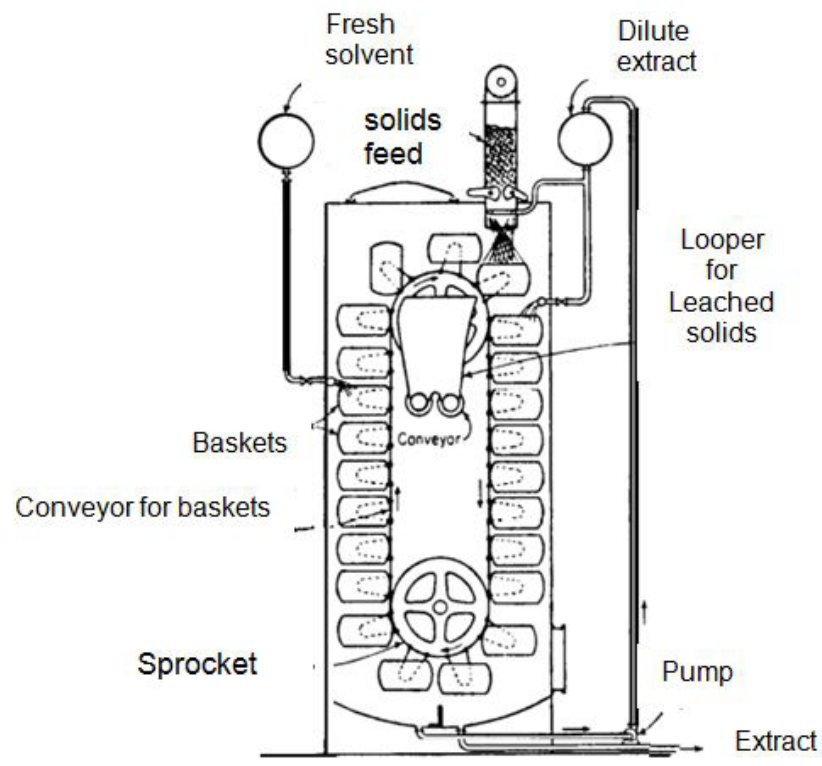

Figura 1. Elevador de cangilones. Adaptado de (Walas, 1990), (Gamse, 2001) y (Jaramillo, 2015).

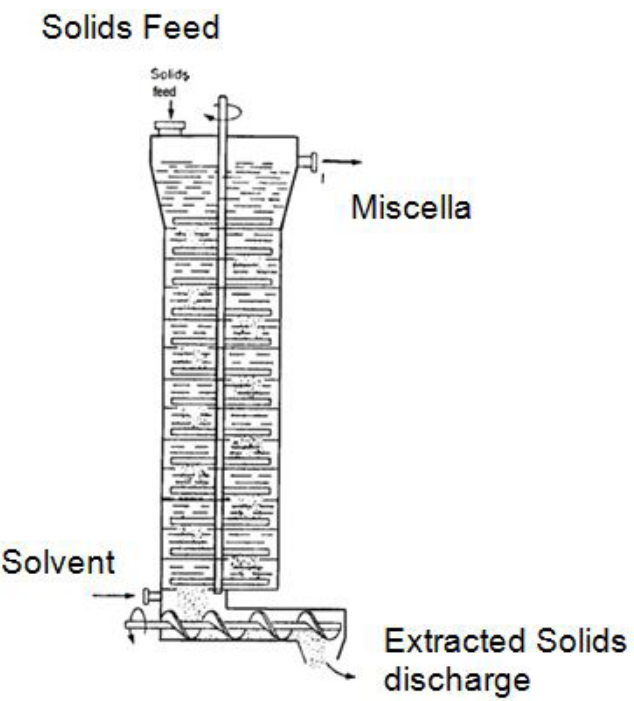

Figura 3. Extractor Tipo Bonotto. Adaptado de (Walas, 1990).

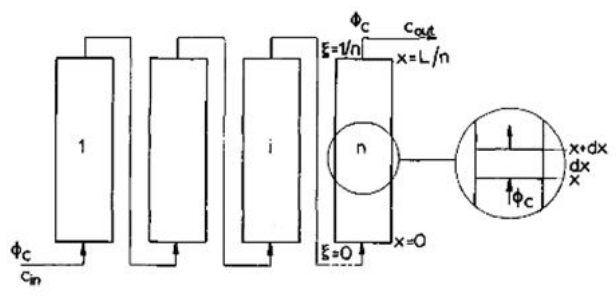

Figura 4. Batería de Difusión Adaptado de (Spaninks, 1979). 


\section{- Diseño de equipos no contínuos}

En estudios a pequeña escala, es viable estudiar el proceso de transferencia de masa entre las fases a través de un contactor intermitente mejor conocido como batería de difusión. Para ello se parte de un modelo de transferencia de masa a partir de la estimación de los coeficientes de transferencia globales en cada fase, generalmente determinados por regresión con la data experimental (Mujica, Delgado, Ramírez, Velásquez, Pérez y Rodriguez-Corella, 2010), (González y Yánez, 2012), (Autor, 2014b).

\section{Aspectos hidráulicos}

\section{- Modelo hidráulico para baterías de difusión}

A continuación se propone un modelo basado en la Ley de Darcy donde se considera que la operación es estable, basándose en el criterio:

$$
\left.\frac{d p}{d z}\right|_{1}=\left.\frac{d p}{d z}\right|_{2}
$$

Considerando la ley de Darcy para estimar la caída de presión, y tomando como referencia un parámetro denominado velocidad critica, definido para determinar la región de estabilidad de la operación la ecuación queda como [4]:

$$
v_{c}=\frac{g\left(\rho_{2}-\rho_{1}\right)}{\frac{\mu_{2}}{k_{2}}-\frac{\mu_{1}}{k_{1}}}
$$

Sin embargo para una operación estable, generalmente se ha determinado una relación lineal entre la caída de presión y el caudal de solvente manejado, para una carga de solido determinada e inmersa dentro de la batería de difusión. No obstante, en operaciones como flujo en lechos empacados, pueden generar inestabilidad, lo cual le brinda mayor complejidad al modelo, ya que debe plantearse un sistema de ecuaciones diferenciales (Walas, 1990), (Gamse, 2001), (Amador y Chavarro, 2012), (Caldas, 2012) y (Jaramillo, 2015).

\section{Otros equipos de extracion solido-liquido}

\section{- Operación discontinua}

\section{Extractor Pot}


Éste tipo de extractor tiene un volumen de 2 a $10 \mathrm{~m} 3$ y un agitador que es requerido para garantizar un mezclado adecuado (Spaninks, 1979), (Walas, 1990), (Gamse, 2001), (Amador y Chavarro, 2012) y (Caldas, 2012).

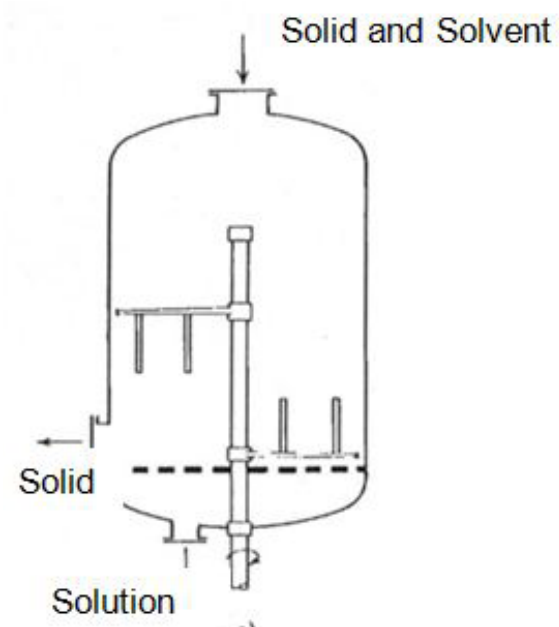

o)

Figura 5. Extractor Pot. Adaptado de (Gamse, 2001).

\section{Extractor rotativo}

Similar al extractor Top en cuanto al mecanismo, sin embargo la posición del equipo en ésta operación es horizontal y cuenta con un sistema de rotación o retromezclado más eficiente (Spaninks, 1979), (Walas, 1990), (Gamse, 2001), (Amador y Chavarro, 2012) y (Caldas, 2012).

Figura 6. Extractor Rotativo Adaptado de (Gamse, 2001).

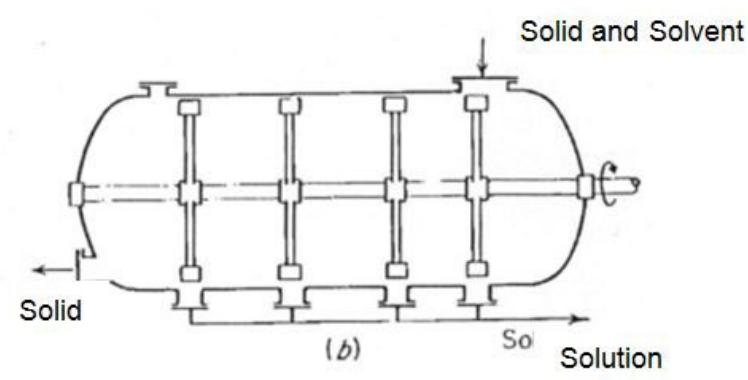

\section{- Operación continua}

\section{Extractor continuo horizontal}

El material sólido se transporta en bandejas y se pone en contacto por percolación con el solvente. El flujo de solvente ingresa al extractor en contracorriente al flujo de sólidos. 


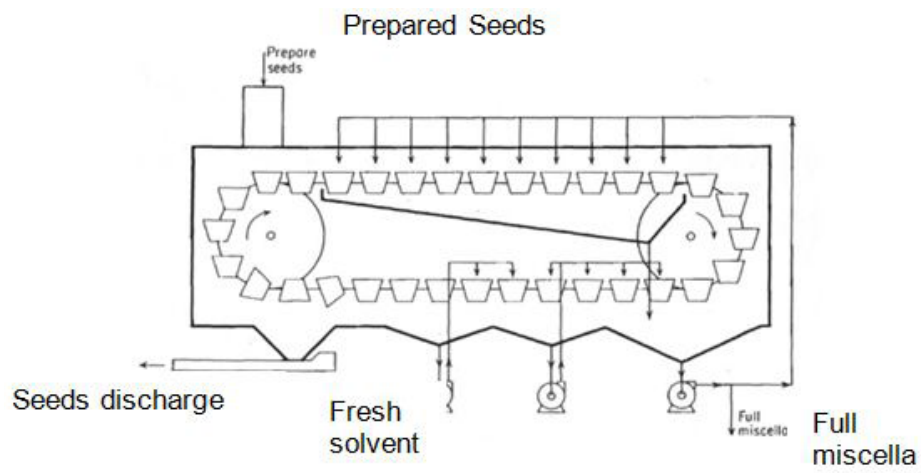

Figura 7. Extractor Continuo Horizontal Adaptado de (Gamse, 2001).

\section{Extractor Kennedy}

La materia prima sólida es transportada por paletas en una cámara donde se mezcla en contracorriente con el solvente. La cámara que se encuentra al final del extractor posee una etapa de filtración para garantizar la pureza del extracto obtenido.

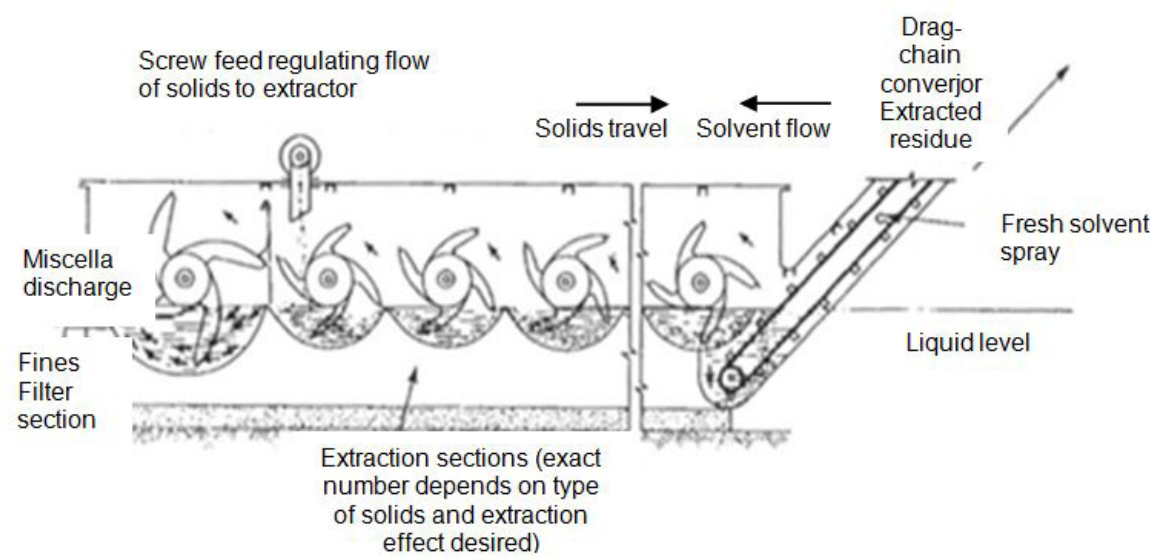

Figura 8. Extractor Kennedy Adaptado de (Gamse, 2001).

\section{Rotocell o extractor tipo carrusel}

Este tipo de extractor opera de tal forma que la materia sólida va girando en torno a un conjunto de compartimientos que van girando continuamente y se ponen en contacto en contracorriente con el solvente, donde se hace circular a éste último empleando para ello bombas. 

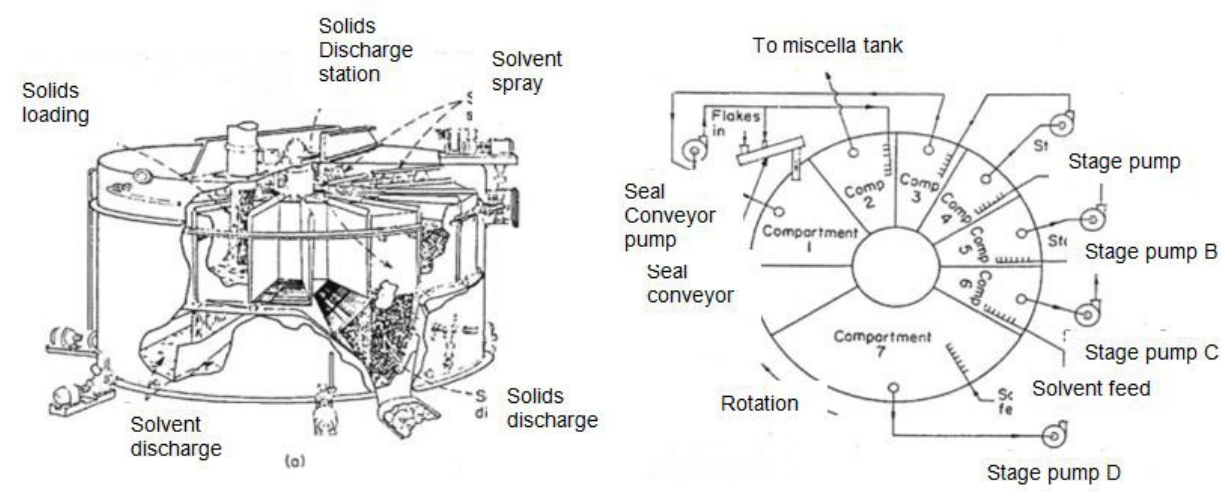

Figura 9. Extractor Rotocell Adaptado de (Gamse, 2001).

\section{Tiempo de residencia}

El tiempo total que el material oleaginoso permanece en el extractor se denomina tiempo de residencia. Éste tiempo puede a su vez subdividirse entre tiempo de "lavado" y tiempo de "drenado". El tiempo de lavado corresponde al retromezclado que ocurre entre el solvente y el material sólido, y el tiempo de drenado es aquel en el cual el material sólido se drena para descargarlo y disponerlo.

Los extractores que poseen un lecho profundo de partículas y una superficie de contacto pequeña generalmente opera sumergiendo el material oleaginoso en el solvente. Éste tipo de operación requiere una gran cantidad de solvente para garantizar un rendimiento considerablemente alto, donde el tiempo de drenado es mucho mayor que el tiempo de lavado.

Los extractores con lechos poco profundos operan por percolación con el solvente, donde ocurre caso contrario, ya que el tiempo de drenado es muy pequeño o despreciable en comparación con el tiempo de contacto.

Generalmente los tiempos de residencia varían entre 30 hasta un máximo de 120 minutos, dependiendo de los requerimientos operacionales y a un balance económico adecuado para garantizar la suficiente rentabilidad (Spaninks, 1979), (Walas, 1990), (Pérez, González, Colón, Morello, Mujica y Martínez, 2009), (Mujica, Delgado, Ramírez, Velásquez, Pérez y Rodriguez-Corella, 2010) y (Jaramillo, 2015).

Torrecilla (2000), emplea un cociente entre el tiempo de residencia $(\tau)$ y tiempo de vida media (el cual corresponde al tiempo promedio que permanece una partícula dentro del lecho). El establece que los valores óptimos se encuentran para valores de $\tau /$ tm inferiores a la unidad. También tenía como premisa el hecho de que existe un tiempo de vida promedio que representaba a ciencia cierta el comportamiento del sólido dentro del sistema, ya que a pesar de que a nivel del modelo real, las 
partículas de una misma carga salen a diferentes intervalos de tiempo, el efecto de alimentación simultánea a la descarga en equipos de contacto continuo, equiparaba de cierta forma ese efecto y podía idealizarse dicho tiempo promedio como tiempo de residencia válido.

\section{Tamaño de partícula}

Una gran variedad de semillas y material oleaginoso es pretratado, donde una de las fases fundamentales de éste proceso lo constituye la molienda. El propósito de la molienda es reducir el tamaño de la partícula del material oleaginoso, con el fin de reducir la distancia o profundidad del lecho de partículas, y por consiguiente el número de extractores requeridos.

Por ejemplo, Kemper (2005), hace referencia al caso particular de las semillas de soya, para un tamaño típico de partícula de $0,38 \mathrm{~mm}$ se requiere un total de 20 extractores. Mediante la reducción del tamaño de partícula, el tiempo que se requiere para que el solvente pueda alcanzar el equilibrio se reduce.

Al reducir el tamaño de la partícula, también se minimiza el tiempo de contacto requerido para lograr extraer cierta cantidad de soluto para un rendimiento determinado. Otro de los parámetros que repercute en reducir el tamaño de la partícula lo constituye el tamaño del extractor, el cual se reduce.

Sin embargo, reducir el tamaño de la partícula representa un costo adicional en el proceso, no sólo a nivel operacional sino también en cuanto a costos energéticos, debido a que se requiere un proceso más riguroso de molienda y tamizado, lo cual redunda en un mayor gasto energético y en equipos más costosos. Por lo tanto, en cada caso particular es requerido llevar a cabo un estudio económico que permita minimizar el costo y maximizar el beneficio, de ese modo se determina el tamaño óptimo de la partícula (Spaninks, 1979), (Bunge, 1981), (Walas, 1990), (Gamse, 2001), (Torrecilla, 2005), (Caldas, 2012), (González y Yánez, 2012), (Autor, 2014b) y (Jaramillo, 2015).

\section{Conclusiones}

Se destacan las siguientes consideraciones finales:

- Las operaciones de extracción sólido-líquido se llevan a cabo en la mayoría de las configuraciones de baterías de equipos de proceso en contracorriente, empleándose bombas para el manejo y transporte del solvente y del lecho fluidizado.

- A escala industrial los equipos más utilizados están constituidos por el extractor tipo cinta perforada, elevador de cangilones y Extractor tipo Bonotto. 
- Para estudios del proceso de extracción a pequeña escala se emplean las denominadas baterías de difusión, las cuales operan de manera intermitente o por carga, sin embargo son de importancia en el estudio del proceso de transferencia de masa y fluodinámica.

- El modelo hidráulico que puede emplearse para modelar las baterías de difusión está representado por el criterio de estabilidad y la ley de Darcy, donde de acuerdo a estudios experimentales previos, generalmente la caída de presión es lineal en función del caudal de solvente, para una carga determinada.

- Entre otros equipos que operan de manera intermitente para la operación de extracción sólido-líquido se encuentran el extractor Pot y el Rotativo.

- Para efectos de otros equipos que operan de manera continua se encuentran el extractor continuo horizontal, tipo Kennedy y Carrusel.

- El tiempo de residencia está determinado por dos componentes: el tiempo de lavado y el tiempo de drenado del solvente, y generalmente se encuentra en el rango de 30 a 120 minutos de extracción.

- El tamaño de la partícula es un aspecto que debe estudiarse particularmente para cada proceso específico, sin embargo casi siempre la reducción del tamaño incide en un mejor desempeño del proceso, sin embargo puede resultar costoso por lo cual es requerido calcular el tamaño óptimo que ofrezca la máxima relación beneficio-costo. 


\section{Referencias}

Durán-García, M. (2014a). "Residual Biomass Gasification: Advances in Bioengineering". Engineering and Applied Sciences: Mathematical and Computational Models. Venezuelan Society of Numerical Methods in Engineering, pp. BSB-7 - BSB-12.

Durán-García, M. (2014b). "Reactores de Lecho Fluidizado y Lecho Burbujeante en la Gasificación de Biomasa Residual". Revista Fuentes, El Reventón Energético, Vol. 12, No 2, pp. 35-43.

Amador, J. y Chavarro, K. (2012). "Revisión de heurísticas y conceptos para el diseño de equipos utilizados en procesos de refinación del petróleo". Trabajo de Grado de Ingeniería Química. Universidad de San Buenaventura, Cartagenas de Indias. pp. 1-80.

Bunge, M (1981). La ciencia, su método y su filosofía. Buenos Aires. Siglo Veinte. $102 \mathrm{pp}$.

Caldas, A. (2012). "Optimización, escalamiento y diseño de una planta piloto de extracción sólido líquido". Trabajo de Grado de Ingeniería Química. Universidad de Cuenca. pp. 1-48.

Gamse, T. (2001). "Extraction". Departamento de Ingeniería Química. Graz University of Technology. " Pág. 20-32.

González, D. y Yánez, Y. (2012). "Diseño y construcción de un extractor sólidolíquido para la obtención de aceite de semillas de sambo y zapallo". Trabajo de Grado de Ingeniería Química. Escuela Superior Politécnica de Chimborazo, Ecuador. pp. 1-110.

Gorrití, A. (2015). "Obtención del aceite vegetal de Euterpe precatoria Mart. (Asaí) por diferentes métodos de extracción: evaluación del rendimiento y calidad (características físico - químicas, actividad antioxidante y estabilidad)". Trabajo de Grado de Maestría en Productos Naturales y Biocomercio, Universidad Nacional Mayor de San Marcos, Perú. pp. 1-134.

Hurtado, J. (2012). El proyecto de Investigación. Caracas. Ediciones Quirón. 191pp.

Jaramillo, M. (2015). "Diseño de una planta piloto para la obtención de $\beta$ metil umbelliferona (4-mu) por microondas mediante la reacción de pechmannduisberg". Trabajo de Grado de Ingeniería Química. Escuela Politécnica Nacional, Ecuador. pp. 1-175. 
Kemper, T. (2005). "Oil extraction". Bailey's Industrial Oil and Fat Products. Sixth Edition. pp. 79-82.

Mujica, V., Delgado, M., Ramírez, M., Velásquez, I., Pérez, C. y RodriguezCorella, M. (2010). "Formulación de un producto cosmético con propiedades antiarrugas a partir del aceite de semilla de merey". Revista de la Facultad de Ingeniería U.C.V., Vol. 25, $\mathrm{N}^{\circ}$ 2, pp. 119-131.

Pérez, C., González, L., Colón, A., Morello, C., Mujica, V. y Martínez, A. (2009). "Evaluación comparativa de los rendimientos obtenidos mediante el proceso de extracción en aceites vegetales a partir de semillas oleaginosas". Revista Anales de la Universidad Metropolitana. Vol. 9, N², pp. 181-206.

Spaninks, J. (1979). "Design procedures for solid-liquid extractors and the effect of hydrodynamic instabilities on extractor performance". Centre for Agricultural Publishing and Documentation. pp. 59-66.

Torrecilla, J. (2005). "Secado del orujo en lecho fluidizado móvil”. Tesis Doctoral. Universidad Complutense de Madrid. pp. 63-64.

Walas, S. (1990). "Chemical process equipment, selection and design". Departamento de Ingeniería Química y Petróleo de la Universidad de Kansas, U.S.A. Primera Edición, pp. 459-493. 
